\title{
ON THE ALGEBRAIC STRINGY EULER NUMBER
}

\author{
VICTOR BATYREV AND GIULIANO GAGLIARDI
}

\begin{abstract}
We are interested in stringy invariants of singular projective algebraic varieties satisfying a strict monotonicity with respect to elementary birational modifications in the Mori program. We conjecture that the algebraic stringy Euler number is one of such invariants. In the present paper, we prove this conjecture for varieties having an action of a connected algebraic group $G$ and admitting equivariant desingularizations with only finitely many $G$-orbits. In particular, we prove our conjecture for arbitrary projective spherical varieties.
\end{abstract}

\section{INTRODUCTION}

Let $X$ be a smooth projective algebraic variety over $\mathbb{C}$. The dimension $b_{\text {top }}^{i}(X)$ of the $i$-th cohomology group $H^{i}(X, \mathbb{C})$ is called the $i$-th topological Betti number of $X$. The topological Betti polynomial of $X$ is defined as

$$
B_{\mathrm{top}}(X, t):=\sum_{i=0}^{2 \operatorname{dim} X}(-1)^{i} b_{\mathrm{top}}^{i}(X) t^{i}
$$

Its value

$$
e_{\mathrm{top}}(X):=B_{\mathrm{top}}(X, 1)=\sum_{i=0}^{2 \operatorname{dim} X}(-1)^{i} b_{\mathrm{top}}^{i}(X)
$$

is the topological Euler number of $X$. Let $H_{\mathrm{alg}}^{2 i}(X, \mathbb{C})$ be the $\mathbb{C}$-subspace in $H^{2 i}(X, \mathbb{C})$ generated by the classes $[Z]$ of algebraic cycles $Z \subseteq X$ of codimension $i$. We call the dimension $b_{\text {alg }}^{2 i}(X)$ of the $\mathbb{C}$-space $H_{\text {alg }}^{2 i}(X, \mathbb{C})$ the $2 i$-th algebraic Betti number of $X$. This naturally leads us to the algebraic Betti polynomial

$$
B_{\mathrm{alg}}(X, t):=\sum_{i=0}^{\operatorname{dim} X} b_{\mathrm{alg}}^{2 i}(X) t^{2 i} .
$$

Its value

$$
e_{\text {alg }}(X):=B_{\text {alg }}(X, 1)=\sum_{i=0}^{\operatorname{dim} X} b_{\text {alg }}^{2 i}(X)
$$

we call the algebraic Euler number of $X$. Using the hard Lefschetz theorem, one can easily show that $e_{\text {alg }}(X) \geq \operatorname{dim} X+1$. In particular, the algebraic Euler number $e_{\text {alg }}(X)$ of a smooth projective variety $X$ is always a positive integer.

In order to consider stringy versions of the above invariants for singular varieties $X$ we need some additional notation.

Let $X$ be a normal projective algebraic variety over $\mathbb{C}$ and let $K_{X}$ be its canonical class. A birational morphism $\rho: Y \rightarrow X$ is called a log-desingularization of $X$ if $Y$ is smooth

2010 Mathematics Subject Classification. Primary 14E30; Secondary 14E15, 14E18, 14L30, 14M27. 
and the exceptional locus of $\rho$ consists of smooth irreducible divisors $D_{1}, \ldots, D_{k}$ with simple normal crossings. Assume that $X$ is $\mathbb{Q}$-Gorenstein, i. e., some integral multiple of $K_{X}$ is a Cartier divisor on $X$. We set $I:=\{1, \ldots, k\}, D_{\emptyset}:=Y$, and for any nonempty subset $J \subseteq I$ we denote by $D_{J}$ the intersection of divisors $\bigcap_{j \in J} D_{j}$, which is either empty or a smooth projective subvariety in $Y$ of codimension $|J|$. Then the canonical classes of $X$ and $Y$ are related by the formula

$$
K_{Y}=\rho^{*} K_{X}+\sum_{i=1}^{k} a_{i} D_{i}
$$

where $a_{1}, \ldots, a_{k}$ are rational numbers which are called discrepancies. The singularities of $X$ are said to be log-terminal if $a_{i}>-1$ for all $i \in I$.

For a projective algebraic variety with at worst $\mathbb{Q}$-Gorenstein log-terminal singularities one defines the topological stringy Betti function

$$
B_{\text {top }}^{\mathrm{str}}(X, t):=\sum_{\emptyset \subseteq J \subseteq I} B_{\mathrm{top}}\left(D_{J}, t\right) \prod_{j \in J}\left(\frac{t^{2}-1}{t^{2\left(a_{j}+1\right)}-1}-1\right)
$$

and the algebraic stringy Betti function

$$
B_{\mathrm{alg}}^{\mathrm{str}}(X, t):=\sum_{\emptyset \subseteq J \subseteq I} B_{\mathrm{alg}}\left(D_{J}, t\right) \prod_{j \in J}\left(\frac{t^{2}-1}{t^{2\left(a_{j}+1\right)}-1}-1\right) .
$$

In particular, one obtains the topological stringy Euler number

$$
e_{\mathrm{top}}^{\mathrm{str}}(X):=\lim _{t \rightarrow 1} B_{\mathrm{top}}^{\mathrm{str}}(X, t)=\sum_{\emptyset \subseteq J \subseteq I} e_{\mathrm{top}}\left(D_{J}\right) \prod_{j \in J}\left(\frac{-a_{j}}{a_{j}+1}\right)
$$

and the algebraic stringy Euler number

$$
e_{\mathrm{alg}}^{\mathrm{str}}(X):=\lim _{t \rightarrow 1} B_{\mathrm{alg}}^{\mathrm{str}}(X, t)=\sum_{\emptyset \subseteq J \subseteq I} e_{\mathrm{alg}}\left(D_{J}\right) \prod_{j \in J}\left(\frac{-a_{j}}{a_{j}+1}\right) .
$$

It is well-known that the definition of $B_{\text {top }}^{\text {str }}(X, t)$ and $e_{\text {top }}^{\text {str }}(X)$ does not depend on the choice of the log-desingularization $\rho$ (see [Bat98]). The proof of this statement uses a non-archimedean integration and the fact that the Betti polynomial $B(X, t)$ can be extended to an additive function on the category $\mathcal{V}_{\mathbb{C}}$ of algebraic varieties over $\mathbb{C}$ having the multiplicativity property

$$
B_{\text {top }}\left(X_{1} \times X_{2}, t\right)=B_{\text {top }}\left(X_{1}, t\right) \cdot B_{\text {top }}\left(X_{2}, t\right) \forall X_{1}, X_{2} \in \mathcal{V}_{\mathbb{C}}
$$

We note that the algebraic Betti polynomial $B_{\text {alg }}(X, t)$ can also be extended to an additive function on the category of arbitrary algebraic varieties, but the above multiplicativity property fails for $B_{\mathrm{alg}}(X, t)$ in general. Nevertheless, it was noticed in Teh09] that the weaker multiplicativity property

$$
B_{\text {alg }}\left(X \times \mathbb{P}^{1}, t\right)=B_{\text {alg }}(X, t) \cdot B_{\text {alg }}\left(\mathbb{P}^{1}, t\right) \forall X \in \mathcal{V}_{\mathbb{C}}
$$

is sufficient in order to define stringy invariants. In particular, the algebraic stringy Betti function $B_{\mathrm{alg}}^{\mathrm{str}}(X, t)$ and the algebraic stringy Euler number $e_{\mathrm{alg}}^{\mathrm{str}}(X)$ do not depend on the choice of the log-desingularization $\rho$. 
The algebraic stringy Euler number $e_{\mathrm{alg}}^{\mathrm{str}}(X)$ of a singular variety $X$ is usually a rational number. Our first conjecture claims the positivity of the algebraic stringy Euler number:

Conjecture 1.1. Let $X$ be a projective algebraic variety with at worst $\mathbb{Q}$-Gorenstein log-terminal singularities. Then $e_{\mathrm{alg}}^{\mathrm{str}}(X)>0$.

Remark 1.2. It is natural to expect an even stronger inequality:

$$
e_{\mathrm{alg}}^{\mathrm{str}}(X) \geq \operatorname{dim} X+1 .
$$

Now we consider some relations between the stringy Euler numbers and birational geometry.

Recall that two smooth birationally isomorphic projective varieties $X_{1}$ and $X_{2}$ are called $K$-equivalent if there exists a birational isomorphism $f: X_{1} \rightarrow X_{2}$ and a smooth projective variety $Y$ together with two birational morphisms $\rho_{1}: Y \rightarrow X_{1}$ and $\rho_{2}: Y \rightarrow X_{2}$ in a commutative diagram

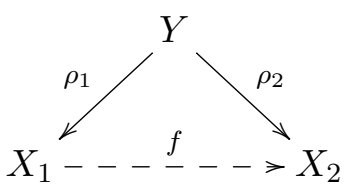

such that the pullbacks of the canonical classes of $X_{1}$ and $X_{2}$ are the same: $\rho_{1}^{*}\left(K_{X_{1}}\right)=$ $\rho_{2}^{*}\left(K_{X_{2}}\right)$. Using methods of non-archimedean integration, one can show that $K$-equivalent algebraic varieties must have the same Betti polynomials: $B_{\text {top }}\left(X_{1}, t\right)=B_{\text {top }}\left(X_{2}, t\right)$. In particular, two birationally isomorphic Calabi-Yau manifolds must have the same Betti numbers (see Bat99a ).

The notion of $K$-equivalence immediately extends to algebraic varieties with at worst $\mathbb{Q}$ Gorenstein singularities. Using the same method based on non-archimedean integration, one can prove that two $K$-equivalent projective varieties $X_{1}$ and $X_{2}$ with at worst log-terminal singularities must have the same stringy Betti functions: $B_{\mathrm{top}}^{\mathrm{str}}\left(X_{1}, t\right)=$ $B_{\mathrm{top}}^{\text {str }}\left(X_{2}, t\right)$. In particular, one has the equality $e_{\mathrm{top}}^{\mathrm{str}}\left(X_{1}\right)=e_{\mathrm{top}}^{\mathrm{str}}\left(X_{2}\right)$ for the stringy Euler numbers if $X_{1}$ and $X_{2}$ are $K$-equivalent.

Definition 1.3. A proper birational morphism $f: X \rightarrow X^{\prime}$ of two $\mathbb{Q}$-Gorenstein varieties $X$ and $X^{\prime}$ is called a divisorial Mori contraction if $f$ contracts a divisor $D \subseteq X$ and the anticanonical divisor $-K_{X}$ is $f$-ample.

Definition 1.4. A birational morphism $g: X \rightarrow X^{+}$of two Q-Gorenstein varieties $X$ and $X^{+}$together with the birational morphisms $f: X \rightarrow Z$ and $f^{+}: X^{+} \rightarrow Z$ in a commutative diagram

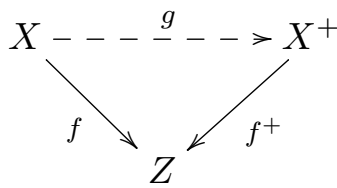

is called a Mori flip if $g$ is an isomorphism in codimension one, the anticanonical class $-K_{X}$ is $f$-ample, and the canonical class $K_{X^{+}}$is $f^{+}$-ample.

We conjecture the following strict monotonicity of the algebraic stringy Euler number with respect to the above elementary birational transformations in the Mori program: 
Conjecture 1.5. Let $f: X \rightarrow X^{\prime}$ be a divisorial Mori contraction. Then

$$
e_{\text {alg }}^{\text {str }}(X)>e_{\text {alg }}^{\text {str }}\left(X^{\prime}\right) .
$$

Conjecture 1.6. Let $g: X \rightarrow X^{+}$be a Mori flip. Then

$$
e_{\text {alg }}^{\mathrm{str}}(X)>e_{\mathrm{alg}}^{\mathrm{str}}\left(X^{+}\right) .
$$

Remark 1.7. From the viewpoint of Conjectures 1.1, 1.5 and 1.6 a projective algebraic variety $X$ with at worst terminal singularities is a minimal model in a given birational class if its algebraic stringy Euler number $e_{\mathrm{alg}}^{\mathrm{str}}(X)$ has the minimal possible value among all projective algebraic varieties with at worst terminal singularities in the same birational class.

Since $e_{\text {top }}(X)=e_{\text {top }}^{\text {str }}(X)$ for smooth algebraic varieties, Conjecture 1.1 is obviously false for the topological stringy Euler number $e_{\text {top }}^{\text {str }}(X)$ because $e_{\text {top }}(C)<0$ already for smooth projective algebraic curves $C$ of genus $g \geq 2$. If $X$ is a blow-up of a smooth curve $C$ in a smooth minimal threefold $X^{\prime}$, then $e_{\text {top }}(X)=e_{\text {top }}\left(X^{\prime}\right)+e_{\text {top }}(C)$ and one gets the opposite inequality $e_{\mathrm{top}}^{\mathrm{str}}(X)<e_{\mathrm{top}}^{\mathrm{str}}\left(X^{\prime}\right)$ if $g \geq 2$. Therefore Conjecture 1.5 is also false for topological stringy Euler numbers. This does not happen for the algebraic Euler number $e_{\text {alg }}(X)$ of smooth projective varieties $X$, because the cohomology groups in odd degrees do not contribute to it.

In general, the topological and algebraic stringy Euler numbers of a singular variety are rational numbers. However, if $X=X_{\Sigma}$ is a $d$-dimensional $\mathbb{Q}$-Gorenstein projective toric variety associated to a rational polyhedral fan $\Sigma$ in a $d$-dimensional $\mathbb{Q}$-space $N_{\mathbb{Q}}$, then the stringy Euler number $e_{\text {top }}^{\text {str }}\left(X_{\Sigma}\right)$ is always a positive integer. This statement follows from the explicit computation of the stringy Betti function of the toric variety $X_{\Sigma}$ by the formula

$$
B_{\text {top }}^{\mathrm{str}}\left(X_{\Sigma}, t\right)=\left(t^{2}-1\right)^{d} \sum_{n \in N} t^{-2 \kappa(n)},
$$

where $N \subseteq N_{\mathbb{Q}}=N \otimes \mathbb{Q}$ is the lattice of 1-parameter subgroups in the $d$-dimensional algebraic torus acting on $X_{\Sigma}$ and $\kappa: N_{\mathbb{Q}} \rightarrow \mathbb{Q}$ is a special piecewise linear function having the following properties: 1) $\kappa$ is linear on every cone $\sigma \in \Sigma ; 2) \kappa$ has value 1 on primitive lattice generators of 1-dimensional cones in $\Sigma$ (see [Bat98]). Moreover, it was observed in [Bat98, Proposition 4.10] that the stringy Euler number $e_{\text {top }}^{\text {str }}\left(X_{\Sigma}\right)$ of a $d$-dimensional toric variety $X_{\Sigma}$ coincides with the normalized volume $d ! \operatorname{vol}(\operatorname{shed}(\Sigma))$ of the bounded polyhedral set

$$
\operatorname{shed}(\Sigma):=\left\{x \in N_{\mathbb{Q}} \mid \kappa(x) \leq 1\right\},
$$

which is called the shed of the fan $\Sigma$ (see [Rei83, (4.2)]). Torus-equivariant birational morphisms of toric varieties $X_{\Sigma} \rightarrow X_{\Sigma^{\prime}}$ can be described by polyhedral subdivisions of the corresponding fans. It was observed by Reid in Rei83 that one has strict inclusions $\operatorname{shed}\left(\Sigma^{\prime}\right) \subsetneq \operatorname{shed}(\Sigma)$ for divisorial toric Mori contractions $f: X_{\Sigma} \rightarrow X_{\Sigma^{\prime}}$ and $\operatorname{shed}\left(\Sigma^{+}\right) \subsetneq \operatorname{shed}(\Sigma)$ for toric Mori flips $g: X_{\Sigma} \rightarrow X_{\Sigma^{+}}$. Therefore, the statements in Conjectures 1.5 and 1.6 hold true for equivariant birational morphisms in the toric Mori program. It is not difficult to show that for projective toric varieties $X$ one always has even the stronger inequality $e_{\mathrm{top}}^{\mathrm{str}}(X) \geq \operatorname{dim} X+1$.

Let $G$ be a connected reductive algebraic group. An irreducible normal $G$-variety $X$ is called spherical if a Borel subgroup $B \subseteq G$ has an open orbit in $X$. Spherical varieties 
are generalizations of toric varieties. Following the equivariant Mori program for toric varieties in [Rei83, the equivariant Mori program for spherical varieties was considered by Brion and Knop in [Bri93, BK94] (see also the recent papers of Perrin and Pasquier Per14, Pas15a]). It is known that a $\mathbb{Q}$-Gorenstein spherical variety is always log-terminal (see [AB04 and [Pas15b, Proposition 5.6]). As in the case of toric varieties, one has the equality $B_{\mathrm{alg}}^{\mathrm{str}}(X, t)=B_{\mathrm{top}}^{\mathrm{str}}(X, t)$ for any spherical variety $X$. However, the stringy Euler number of a projective spherical variety $X$ is not always an integer even in the case when $X$ is Gorenstein (see [BM13, Example 4.6]).

Our main purpose of this paper is to attract attention to the above conjectures and to prove them for arbitrary projective spherical varieties:

Theorem 1.8. Let $G$ be a connected reductive algebraic group. Conjectures 1.1, 1.5 and 1.6 are true in the $G$-equivariant Mori program for projective spherical G-varieties.

We remark that our proof of the above theorem works even for more general $G$-varieties having a regular action of a connected algebraic group $G$. We need only the following well-known properties of spherical varieties: 1) every spherical $G$-variety $X$ contains only finitely many $G$-orbits; 2 ) every singular spherical $G$-variety $X$ always admits a $G$ equivariant log-desingularization $Y$ which is again a spherical variety; 3 ) every birational $G$-equivariant map $g: X_{1} \rightarrow X_{2}$ between two spherical $G$-varieties can be extended to a $G$-equivariant commutative diagram

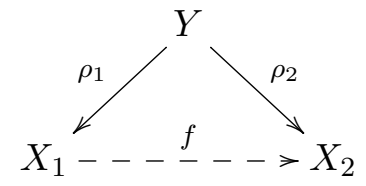

for some smooth spherical $G$-variety $Y$ and $G$-equivariant log-desingularizations $\rho_{1}: Y \rightarrow$ $X_{1}$ and $\rho_{2}: Y \rightarrow X_{2}$.

In Section 2, we explain the construction of generalized stringy invariants of algebraic varieties that include the algebraic stringy Euler number and some other invariants that could attain minimum on the minimal models in a given birational class.

In Section 3, we prove Theorem 1.8 as a consequence of some more general results on the $G$-equivariant birational Mori theory. We also discuss some generalizations of our results that include the $G$-equivariant Mori program for pairs $(X, \Delta)$.

\section{Generalized stringy invariants of algebraic varieties}

We denote by $\mathcal{V}_{\mathbb{C}}$ the category of complex algebraic varieties. Let $K_{0}\left(\mathcal{V}_{\mathbb{C}}\right)$ be the Grothendieck group of complex algebraic varieties, which is the quotient of the free abelian group generated by isomorphism classes $[X]$ of complex algebraic varieties modulo the subgroup generated by $[X]-\left[X^{\prime}\right]-\left[X \backslash X^{\prime}\right]$ for closed subvarieties $X^{\prime} \subseteq X$. One defines the structure of a commutative ring on $K_{0}\left(\mathcal{V}_{\mathbb{C}}\right)$ by the equation $[X] \cdot\left[X^{\prime}\right]:=\left[X \times X^{\prime}\right]$. We can consider the ring $K_{0}\left(\mathcal{V}_{\mathbb{C}}\right)$ as a module over the polynomial ring $\mathbb{Z}[\lambda]$ using the ring homomorphism $\mathbb{Z}[\lambda] \rightarrow K_{0}\left(\mathcal{V}_{\mathbb{C}}\right)$ that maps $\lambda$ to the class $\mathbb{L}:=\left[\mathbb{A}^{1}\right] \in K_{0}\left(\mathcal{V}_{\mathbb{C}}\right)$.

Let $S:=S(\lambda) \subseteq \mathbb{Z}[\lambda]$ be the multiplicative subset generated by $\lambda$ and the polynomials $\sum_{i=0}^{k} \lambda^{i}$ for all $k \geq 0$. We define a filtration $F_{\infty}^{*}$ on the localized ring $S^{-1} \mathbb{Z}[\lambda]$ using the subgroups $F_{\infty}^{m} S^{-1} \mathbb{Z}[\lambda](m \in \mathbb{Z})$ generated by all rational functions $p(\lambda) / q(\lambda)$ such that $q(\lambda) \in S$ and $\operatorname{deg} q-\operatorname{deg} p \geq m$. This filtration is obtained by the restriction to 
$S^{-1} \mathbb{Z}[\lambda]$ of the filtration on the ring of rational functions on $\mathbb{P}^{1}$ over Spec $\mathbb{Z}$ defined by the discrete valuation corresponding to the point $\infty \in \mathbb{P}^{1}$.

We define the filtration $\mathcal{F}^{*}$ on the $S^{-1} \mathbb{Z}[\lambda]$-algebra

$$
\widetilde{\mathcal{M}}_{\mathbb{C}}:=S^{-1} K_{0}\left(\mathcal{V}_{\mathbb{C}}\right)
$$

where $\mathcal{F}^{m} \widetilde{\mathcal{M}}_{\mathbb{C}}$ is the subgroup of $\widetilde{\mathcal{M}}_{\mathbb{C}}$ generated by

$$
\left\{\frac{[X]}{q(\mathbb{L})}: \operatorname{deg} q(\lambda)-\operatorname{dim} X \geq m, q(\lambda) \in S\right\} .
$$

This filtration is compatible with the filtration $F_{\infty}^{*}$ on $S^{-1} \mathbb{Z}[\lambda]$ :

$$
F_{\infty}^{m} \cdot \mathcal{F}^{l} \subseteq \mathcal{F}^{m+l} \quad \forall m, l \in \mathbb{Z} .
$$

In papers devoted to motivic integration (see, for instance, [DL99, (3.2)] or [Cra04, Definition 2.11]) one often considers the ring $\widehat{\mathcal{M}}_{\mathbb{C}}$ obtained as the completion of the ring $\widetilde{\mathcal{M}}_{\mathbb{C}}$ with respect to the filtration $\mathcal{F}^{*}$. However, the kernel of the completion map $\widetilde{\mathcal{M}}_{\mathbb{C}} \rightarrow \widehat{\mathcal{M}}_{\mathbb{C}}$ is rather difficult to understand because $\widetilde{\mathcal{M}}_{\mathbb{C}}$ is not a noetherian ring. The above filtration is necessary for the definition of the topology and the convergence in this topology, but we do not really need the completed ring $\widehat{\mathcal{M}}_{\mathbb{C}}$ because all non-archimedean motivic integrals considered will take their values in the localized ring $\widetilde{\mathcal{M}}_{\mathbb{C}}$ or in an extension $\widetilde{\mathcal{M}}_{\mathbb{C}}^{(r)}$, which we explain next.

Let $r$ be a positive integer. Using the ring monomorphism

$$
\imath_{r}: S^{-1} \mathbb{Z}[\lambda] \rightarrow S^{-1} \mathbb{Z}[\lambda], \quad \lambda \mapsto \lambda^{r},
$$

we can identify the image of $\imath_{r}$ with $S^{-1} \mathbb{Z}[\lambda]$ and obtain the ring extension

$$
S^{-1} \mathbb{Z}[\lambda] \subseteq S\left(\lambda^{1 / r}\right)^{-1} \mathbb{Z}\left[\lambda^{1 / r}\right]
$$

This allows us to consider any rational function

$$
\frac{\lambda-1}{\lambda^{k / r}-1}, \quad k \in \mathbb{N}
$$

as an element of $S\left(\lambda^{1 / r}\right)^{-1} \mathbb{Z}\left[\lambda^{1 / r}\right]$. For any $\mathbb{Z}[\lambda]$-module $M$, we define

$$
S_{(r)}^{-1} M:=S\left(\lambda^{1 / r}\right)^{-1} \mathbb{Z}\left[\lambda^{1 / r}\right] \otimes_{S^{-1}} \mathbb{Z}[\lambda] S^{-1} M,
$$

and we define

$$
\widetilde{\mathcal{M}}_{\mathbb{C}}^{(r)}:=S_{(r)}^{-1} K_{0}\left(\mathcal{V}_{\mathbb{C}}\right)
$$

The following Definition 2.1 and Theorem 2.2 are slightly modified versions of some of the content from [Teh09, Section 4].

Definition 2.1. Let $X$ be a normal projective variety over $\mathbb{C}$ with at worst $\mathbb{Q}$-Gorenstein log-terminal singularities. Denote by $r$ the minimal positive integer such that $r K_{X}$ is a Cartier divisor. Let $\rho: Y \rightarrow X$ be a log-desingularization together with smooth irreducible divisors $D_{1}, \ldots, D_{k}$ with simple normal crossings whose support covers the exceptional locus of $\rho$. We can uniquely write

$$
K_{Y}=\rho^{*} K_{X}+\sum_{i=1}^{k} a_{i} D_{i}
$$


for some rational numbers $a_{i} \in \frac{1}{r} \mathbb{Z}$ satisfying the additional condition $a_{i}=0$ if $D_{i}$ is not in the exceptional locus of $\rho$. We set $I:=\{1, \ldots, k\}$ and, for any $\emptyset \subseteq J \subseteq I$, we define

$$
D_{J}:=\left\{\begin{array}{ll}
Y & \text { if } J=\emptyset, \\
\bigcap_{j \in J} D_{j} & \text { if } J \neq \emptyset,
\end{array} \quad D_{J}^{\circ}:=D_{J} \backslash \bigcup_{j \in I \backslash J} D_{j} .\right.
$$

Let $\phi: K_{0}\left(\mathcal{V}_{\mathbb{C}}\right) \rightarrow M$ be a $\mathbb{Z}[\lambda]$-module homomorphism. It induces a $S_{(r)}^{-1} \mathbb{Z}[\lambda]$-module homomorphism

$$
S_{(r)}^{-1} \phi: \widetilde{\mathcal{M}}_{\mathbb{C}}^{(r)} \rightarrow S_{(r)}^{-1} M
$$

We define the generalized stringy invariant associated to $\phi$ as

$$
\begin{aligned}
\phi^{\operatorname{str}}(X) & :=\sum_{\emptyset \subseteq J \subseteq I}\left(\prod_{j \in J} \frac{\lambda-1}{\lambda^{a_{j}+1}-1}\right) \cdot \phi\left(D_{J}^{\circ}\right) \\
& =\sum_{\emptyset \subseteq J \subseteq I}\left(\prod_{j \in J} \frac{\lambda-1}{\lambda^{a_{j}+1}-1}-1\right) \cdot \phi\left(D_{J}\right) \in S_{(r)}^{-1} M
\end{aligned}
$$

Theorem 2.2. The definition of $\phi^{\mathrm{str}}(X)$ is independent of the choice of the log-desingularization.

Proof. It follows from the theory of motivic integration (see, for instance, Vey01, Appendix]) that the element

$$
[X]^{\mathrm{str}}:=\sum_{\emptyset \subseteq J \subseteq I}\left(\prod_{j \in J} \frac{\mathbb{L}-1}{\mathrm{~L}^{a_{j}+1}-1}\right) \cdot\left[D_{J}^{\circ}\right] \in \widetilde{\mathcal{M}}_{\mathbb{C}}^{(r)}
$$

is independent of the choice of the log-desingularization. By $\phi^{\operatorname{str}}(X)=S_{(r)}^{-1} \phi\left([X]^{\text {str }}\right)$, the element $\phi^{\operatorname{str}}(X)$ is also independent of the choice of the log-desingularization.

We now consider examples of $\mathbb{Z}[\lambda]$-module homomorphisms $\phi: K_{0}\left(\mathcal{V}_{\mathbb{C}}\right) \rightarrow M$ and their respective stringy invariants.

Example 2.3. The virtual Hodge polynomial (also known as the E-polynomial)

$$
E(X ; u, v):=\sum_{p, q}(-1)^{p+q} h^{p, q}(X) u^{p} v^{q}
$$

induces a $\mathbb{Z}[\lambda]$-module homomorphism $K_{0}\left(\mathcal{V}_{\mathbb{C}}\right) \rightarrow \mathbb{Z}[u, v]$ where the $\mathbb{Z}[\lambda]$-module structure on $\mathbb{Z}[u, v]$ is given by the map $\lambda \mapsto u v$. By Theorem 2.2 we obtain the stringy E-function

$$
E^{\operatorname{str}}(X ; u, v) \in S_{(r)}^{-1} \mathbb{Z}[u, v] .
$$

Example 2.4. The topological virtual Betti polynomial

$$
B_{\text {top }}(X ; t):=E(X ; t, t)
$$

induces a $\mathbb{Z}[\lambda]$-module homomorphism $K_{0}\left(\mathcal{V}_{\mathbb{C}}\right) \rightarrow \mathbb{Z}[t]$ where the $\mathbb{Z}[\lambda]$-module structure on $\mathbb{Z}[t]$ is given by the map $\lambda \mapsto t^{2}$. By Theorem 2.2 , we obtain the topological stringy Betti function

$$
B_{\text {top }}^{\text {str }}(X ; t) \in S_{(r)}^{-1} \mathbb{Z}\left[t^{2}\right] .
$$


Example 2.5. The topological Euler number

$$
e_{\text {top }}(X):=B(X ; 1)=E(X ; 1,1)
$$

induces a $\mathbb{Z}[\lambda]$-module homomorphism $K_{0}\left(\mathcal{V}_{\mathbb{C}}\right) \rightarrow \mathbb{Z}$ where the $\mathbb{Z}[\lambda]$-module structure on $\mathbb{Z}$ is given by the map $\lambda \mapsto 1$. By Theorem 2.2 , we obtain the topological stringy Euler number

$$
e_{\text {top }}^{\text {str }}(X) \in S_{(r)}^{-1} \mathbb{Z}
$$

Using the map $S_{(r)}^{-1} \mathbb{Z} \rightarrow \mathbb{Q}, \lambda^{1 / r} \mapsto 1$, we interpret $e_{\text {top }}^{\text {str }}(X) \in \mathbb{Q}$, and then we have

$$
e_{\mathrm{top}}^{\mathrm{str}}(X)=\lim _{t \rightarrow 1} B_{\mathrm{top}}^{\mathrm{str}}(X ; t)=\lim _{u, v \rightarrow 1} E^{\mathrm{str}}(X ; u, v) .
$$

The following was shown by Teh using [Bit04, Theorem 3.1].

Proposition 2.6 ([Teh09, Proposition $4, k=2 p]$ ). The algebraic Betti polynomial of smooth projective algebraic varieties

$$
B_{\mathrm{alg}}(X ; t):=\sum_{i} b_{\mathrm{alg}}^{2 i}(X) t^{2 i}
$$

can be extended to a $\mathbb{Z}[\lambda]$-module homomorphism

$$
B_{\text {alg }}: K_{0}\left(\mathcal{V}_{\mathbb{C}}\right) \rightarrow \mathbb{Z}\left[t^{2}\right]
$$

where the $\mathbb{Z}[\lambda]$-module structure on $\mathbb{Z}\left[t^{2}\right]$ is given by $\lambda \mapsto t^{2}$.

Corollary 2.7. By Theorem 2.2, we obtain that the algebraic stringy Betti function

$$
B_{\mathrm{alg}}^{\mathrm{str}}(X ; t) \in S_{(r)}^{-1} \mathbb{Z}[t]
$$

does not depend on the choice of the log-desingularization and the same is true for the algebraic stringy Euler number

$$
e_{\text {alg }}^{\mathrm{str}}(X)=\lim _{t \rightarrow 1} B_{\mathrm{alg}}^{\mathrm{str}}(X ; t) \in \mathbb{Q} .
$$

Example 2.8. It is also possible to consider the following modified version of the virtual E-polynomial:

$$
E_{\text {even }}(X ; u, v):=\frac{1}{2}(E(X ; u, v)+E(X ;-u,-v))=\sum_{p+q \in 2 \mathbb{Z}} h^{p, q}(X) u^{p} v^{q} .
$$

The corresponding stringy invariant

$$
E_{\text {even }}^{\mathrm{str}}(X ; u, v):=\sum_{\emptyset \subseteq J \subseteq I} E_{\text {even }}\left(D_{J}^{\circ} ; u, v\right)\left(\prod_{j \in J} \frac{u v-1}{(u v)^{a_{j}+1}-1}\right)
$$

we call the even stringy E-function.

Example 2.9. Another modified version of the virtual $E$-polynomial is

$$
E_{p=q}(X ; u, v):=\sum_{0 \leq p \leq \operatorname{dim} X} h^{p, p}(X) u^{p} v^{p} .
$$

This leads to the stringy invariant

$$
E_{p=q}^{\mathrm{str}}(X ; u, v):=\sum_{\emptyset \subseteq J \subseteq I} E_{p=q}\left(D_{J}^{\circ} ; u, v\right)\left(\prod_{j \in J} \frac{u v-1}{(u v)^{a_{j}+1}-1}\right) .
$$


Remark 2.10. We also obtain the modified Euler numbers

$$
e_{\mathrm{even}}^{\mathrm{str}}(X):=\lim _{u, v \rightarrow 1} E_{\text {even }}^{\mathrm{str}}(X ; u, v) \quad \text { and } \quad e_{p=q}^{\mathrm{str}}(X):=\lim _{u, v \rightarrow 1} E_{p=q}^{\mathrm{str}}(X ; u, v) .
$$

The results of the next section show that one can expect that Conjectures 1.1, 1.5, and 1.6 could also be true for the rational numbers $e_{\text {even }}^{\text {str }}$ and $e_{p=q}^{\text {str }}$, i. e., these alternative stringy invariants are also candidates for invariants attaining their minimum on the minimal model in a given birational class.

\section{EQUIVARIANT DESINGULARIZATIONS WITH FINITELY MANY ORBITS}

Let $G$ be a connected linear algebraic group. We consider the category of algebraic $G$-varieties with $G$-equivariant birational maps over $\mathbb{C}$. We need two results of Brion and Peyre.

Lemma 3.1 ([BP02, Lemma 1 and Lemma 2]). Let $H \subseteq G$ be a closed subgroup. Then there exists a locally trivial fibration in the Zariski topology $G / H \rightarrow Z$ with fiber $T$ such that $B_{\mathrm{alg}}(Z ; t)=B_{\mathrm{top}}(Z ; t)$ and $T$ is an algebraic torus.

Proposition 3.2. Let $H \subseteq G$ be a closed subgroup. Then for the quasi-projective variety $G / H$ one has

$$
B_{\text {alg }}(G / H ; t)=B_{\text {top }}(G / H ; t) .
$$

In particular, one has $e_{\text {alg }}(G / H)=e_{\text {top }}(G / H)$.

Proof. Using the fibration from Lemma 3.1, one obtains

$$
B_{\text {alg }}(G / H ; t)=B_{\text {alg }}(T ; t) B_{\text {alg }}(Z ; t)=B_{\text {top }}(T ; t) B_{\text {top }}(Z ; t)=B_{\text {top }}(G / H ; t)
$$

because $B_{\text {alg }}(T ; t)=B_{\text {top }}(T ; t)=\left(t^{2}-1\right)^{\operatorname{dim} T}$.

Proposition 3.3 ([ВP02, Theorem 1(b)]). Let $H \subseteq G$ be a closed subgroup. Then one has

$$
e_{\text {top }}(G / H)= \begin{cases}0 & \text { if } \operatorname{rank} G>\operatorname{rank} H, \\ \left|W_{G}\right| /\left|W_{H}\right| & \text { otherwise, }\end{cases}
$$

where $W_{G}$ (resp. $\left.W_{H}\right)$ denotes the Weyl group of $G^{\mathrm{red}}$ (resp. of $\left.\left(H^{\circ}\right)^{\mathrm{red}}\right)$. In particular, one has $e_{\mathrm{top}}(G / H)>0$ if $H$ is a parabolic subgroup.

Theorem 3.4. Let $X$ be a $\mathbb{Q}$-Gorenstein projective $G$-variety with at worst log-terminal singularities. Assume that there exists a $G$-equivariant log-desingularization $\rho: Y \rightarrow X$ such that $Y$ contains only finitely many $G$-orbits. Then $e_{\text {alg }}^{\text {str }}(X)>0$.

Proof. We write

$$
K_{Y}=\rho^{*} K_{X}+\sum_{i=1}^{k} a_{i} D_{i}
$$

for some $G$-invariant smooth irreducible divisors $D_{1}, \ldots, D_{k}$. By Definition 2.1, we have

$$
e_{\mathrm{alg}}^{\mathrm{str}}(X)=\sum_{\emptyset \subseteq J \subseteq I} e_{\mathrm{alg}}\left(D_{J}^{\circ}\right) \prod_{j \in J} \frac{1}{a_{j}+1} .
$$

Since the $G$-invariant locally closed subvariety $D_{J}^{\circ}$ is a union of finitely many $G$-orbits, by Proposition 3.3. we obtain $e_{\text {alg }}\left(D_{J}^{\circ}\right) \geq 0$ for all $J \subseteq I$. Together with the inequalities 
$a_{j}+1>0$ for all $j \in I$ it implies $e_{\text {alg }}^{\text {str }}(X) \geq 0$. Choose a subset $J \subseteq I$ with $D_{J}^{\circ}=D_{J} \neq \emptyset$. As $D_{J}^{\circ}$ contains a projective $G$-orbit, by Proposition 3.3 , we obtain $e_{\text {alg }}\left(D_{J}^{\circ}\right)>0$ and, hence, the strict inequality $e_{\text {alg }}^{\mathrm{str}}(X)>0$.

Theorem 3.5. Let $g: X \rightarrow X^{+}$be a G-equivariant Mori flip with G-equivariant logdesingularizations $\rho: Y \rightarrow X$ and $\rho_{+}: Y \rightarrow X^{+}$as well as a $G$-equivariant commutative diagram

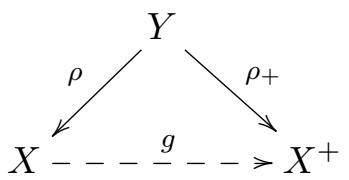

such that $Y$ contains only finitely many $G$-orbits. Then we have $e_{\mathrm{alg}}^{\mathrm{str}}(X)>e_{\mathrm{alg}}^{\mathrm{str}}\left(X^{+}\right)$.

Proof. Write

$$
K_{Y}=\rho^{*} K_{X}+\sum_{i=1}^{k} a_{i} D_{i}=\rho_{+}^{*} K_{X^{+}}+\sum_{i=1}^{k} a_{i}^{+} D_{i}
$$

for some $G$-invariant smooth irreducible divisors $D_{1}, \ldots, D_{k}$. By Definition 2.1, we have

$$
\begin{aligned}
e_{\mathrm{alg}}^{\mathrm{str}}(X) & =\sum_{\emptyset \subseteq J \subseteq I} e_{\mathrm{alg}}\left(D_{J}^{\circ}\right) \prod_{j \in J} \frac{1}{a_{j}+1}, \\
e_{\mathrm{alg}}^{\mathrm{str}}\left(X^{+}\right) & =\sum_{\emptyset \subseteq J \subseteq I} e_{\mathrm{alg}}\left(D_{J}^{\circ}\right) \prod_{j \in J} \frac{1}{a_{j}^{+}+1} .
\end{aligned}
$$

According to [IS05, Lemma 3.4], we have $a_{j} \leq a_{j}^{+}$for every $j \in I$ and there exists $j_{0} \in I$ such that $a_{j_{0}}<a_{j_{0}}^{+}$. Since the $G$-invariant locally closed subvariety $D_{J}^{\circ}$ is a union of finitely many $G$-orbits, by Proposition 3.3 , we have $e_{\text {alg }}\left(D_{J}^{\circ}\right) \geq 0$ for all subsets $J \subseteq I$. Thus, we obtain $e_{\text {alg }}^{\text {str }}(X) \geq e_{\text {alg }}^{\text {str }}\left(X^{+}\right)$. Choose a subset $J \subseteq I$ with $j_{0} \in J$ and $D_{J}^{\circ}=D_{J} \neq \emptyset$. As $D_{J}^{\circ}$ contains a projective $G$-orbit, by Proposition 3.3 , we obtain $e_{\mathrm{alg}}\left(D_{J}^{\circ}\right)>0$ and, hence, the strict inequality $e_{\mathrm{alg}}^{\mathrm{str}}\left(X^{-}\right)>e_{\mathrm{alg}}^{\mathrm{str}}\left(X^{+}\right)$.

Theorem 3.6. Let $f: X \rightarrow X^{\prime}$ be a G-equivariant divisorial Mori contraction with a $G$-equivariant log-desingularization $\rho: Y \rightarrow X$ such that $Y$ contains only finitely many $G$-orbits. Then $e_{\mathrm{str}}^{\operatorname{alg}}(X)>e_{\mathrm{str}}^{\mathrm{alg}}\left(X^{\prime}\right)$.

Proof. We write

$$
K_{Y}=\rho^{*}\left(K_{X}\right)+\sum_{i=1}^{k} a_{i} D_{i}=\rho^{*} f^{*} K_{X^{\prime}}+\sum_{i=1}^{k} a_{i}^{\prime} D_{i}
$$

for some $G$-invariant smooth irreducible divisors $D_{1}, \ldots, D_{k}$. By Definition 2.1, we have

$$
\begin{aligned}
& e_{\mathrm{alg}}^{\mathrm{str}}(X)=\sum_{\emptyset \subseteq J \subseteq I} e_{\mathrm{alg}}\left(D_{J}^{\circ}\right) \prod_{j \in J} \frac{1}{a_{j}+1}, \\
& e_{\mathrm{alg}}^{\mathrm{str}}\left(X^{\prime}\right)=\sum_{\emptyset \subseteq J \subseteq I} e_{\mathrm{alg}}\left(D_{J}^{\circ}\right) \prod_{j \in J} \frac{1}{a_{j}^{\prime}+1} .
\end{aligned}
$$

Again we have $a_{j} \leq a_{j}^{\prime}$ for every $j \in I$ and there exists $j_{0} \in I$ such that $a_{j_{0}}<a_{j_{0}}^{\prime}$. The rest of the proof is the same as in the proof of Theorem 3.5 . 
Remark 3.7 (Proof of Theorem 1.8). Spherical varieties have only finitely many $G$-orbits (see, for instance, [Per14, Theorem 2.1.2]). Moreover, the desingularizations of spherical varieties required in Theorems 3.4 , 3.5 and 3.6 can be always constructed explicitly using subdivisions of the corresponding colored fans (see, for instance, [Per14, Theorem 3.1.13 and Corollary 3.3.8]).

Remark 3.8. As in Bat99b], it is possible to generalize Definition 2.1 to define stringy invariants for any Kawamata log-terminal pair $(X, \Delta)$.

For example, let $\rho: Y \rightarrow X$ be a log-desingularization of $(X, \Delta)$ together with smooth irreducible divisors $D_{1}, \ldots, D_{k}$ with simple normal crossings whose support covers the exceptional locus of $\rho$ and the support of $\rho^{*} \Delta$. We can uniquely write

$$
K_{Y}=\rho^{*}\left(K_{X}+\Delta\right)+\sum_{i=1}^{k} a_{i} D_{i}
$$

for some rational numbers $a_{i} \in \frac{1}{r} \mathbb{Z}$ satisfying the additional condition that $-a_{i}$ is the multiplicity of $D_{i}$ in $\Delta$ if $D_{i}$ is not in the exceptional locus of $\rho$. We then define

$$
e_{\mathrm{alg}}^{\mathrm{str}}(X, \Delta):=\sum_{\emptyset \subseteq J \subseteq I} e_{\mathrm{alg}}^{\mathrm{str}}\left(D_{j}^{\circ}\right) \prod_{j \in J} \frac{1}{a_{j}+1} \in \mathbb{Q} .
$$

Conjecture 3.9. The algebraic stringy Euler number $e_{\mathrm{alg}}^{\mathrm{str}}(X, \Delta)$ is strictly monotone with respect to the elementary birational transformations from the Mori theory of Kawamata log-terminal pairs $(X, \Delta)$.

It is possible to prove generalized versions of Theorems 3.4, 3.5 and 3.6 for Kawamata log-terminal pairs $(X, \Delta)$ where the irreducible components of $\Delta$ are $G$-invariant. In particular, we obtain the following result.

Theorem 3.10. The algebraic stringy Euler number $e_{\mathrm{alg}}^{\mathrm{str}}(X, \Delta)$ is strictly monotone with respect to the elementary $G$-equivariant birational transformations from the $G$-equivariant Mori theory of Kawamata log-terminal pairs $(X, \Delta)$ for projective spherical varieties $X$ and $G$-invariant divisors $\Delta$.

Remark 3.11. Let $B \subseteq G$ be a Borel subgroup. Since every divisor on a spherical variety is linearly equivalent to a $B$-invariant divisor, it is natural to consider the $B$-equivariant Mori theory of pairs $(X, \Delta)$ on spherical $G$-varieties $X$. Irreducible $B$-invariant divisors on $X$ that are not $G$-invariant are called colors. As in the case of toric varieties, $B$-invariant Cartier divisors can be described by piecewise linear functions on the colored fan of a spherical variety. It is possible that the irreducible $B$-invariant divisors (colors) have singularities, even in the open $G$-orbit, and even if all colors are smooth, they may not satisfy the normal crossing condition. The $B$-equivariant log-desingularizations of $B$-invariant divisors on horospherical varieties have been constructed by Pasquier in [Pas16] using Bott-Samelson resolutions of Schubert cells in homogeneous spaces. We remark that every spherical variety $X$ contains only finitely many $B$-orbits. However, a $B$-equivariant log-desingularization of $B$-invariant pairs on a spherical variety $X$ having only finitely many $B$-orbits may not exist in general.

Proposition 3.12. Consider the spherical $\mathrm{SL}(2)$-variety $X:=\mathbb{P}\left(\mathfrak{s l}_{2}\right) \cong \mathbb{P}^{2}$, i. e., the projectivization of the adjoint representation of $\mathrm{SL}(2)$. Then the set of B-invariant 
divisors in $X$ does not admit a B-equivariant log-desingularization with finitely many B-orbits.

Proof. It is easy to show that $X$ contains exactly two irreducible $B$-invariant divisors $D_{0}$ and $D_{1}$. The divisor $D_{0} \cong \mathbb{P}^{1}$ is an $\mathrm{SL}(2)$-invariant conic in $\mathbb{P}^{2}$. It is the projectivization of the set of nilpotent matrices in $\mathfrak{s l}_{2}$. The $B$-invariant divisor $D_{1} \cong \mathbb{P}^{1}$ is the tangent line to the conic $D_{0}$ at the $B$-fixed point $x_{B} \in D_{0}$.

Assume that there exists a $B$-equivariant desingularization $\rho: Y \rightarrow X$ such that $Y$ has only finitely many $B$-orbits and the set of $B$-invariant divisors in $Y$ has simple normal crossings. Then the birational morphism $\rho: Y \rightarrow X$ between two smooth projective algebraic surfaces $Y$ and $X$ can be decomposed into a sequence of $B$-equivariant blowdowns $Y=: X_{n} \rightarrow X_{n-1} \rightarrow \cdots \rightarrow X_{2} \rightarrow X_{1} \rightarrow X_{0}:=X$. Moreover, every smooth projective algebraic surface $X_{i}$ must have finitely many $B$-orbits. Since $X_{0}$ has only one $B$-fixed point $x_{0}:=D_{0} \cap D_{1}$, the birational morphism $\rho_{1}: X_{1} \rightarrow X_{0}$ must be the blow-up of this point. Then the $B$-variety $X_{1}$ contains three $B$-invariant divisors $\rho^{-1}\left(D_{0}\right)$, $\rho^{-1}\left(D_{1}\right)$, and $\rho^{-1}\left(x_{0}\right)$ having a common $B$-fixed point $x_{1} \in X_{1}$. It is easy to show that $x_{1}$ is the unique $B$-fixed point in $X_{1}$. Therefore, the birational $B$-equivariant morphism $\rho_{2}: X_{2} \rightarrow X_{1}$ must be the blow-up of $x_{1}$. On the other hand, the tangent spaces of three divisors at the point $x_{1}$ are pairwise different. Hence, the group $B$ has at least 3 pairwise distinct fixed points in the exceptional divisor $E:=\rho^{-1}\left(x_{1}\right) \subseteq X_{2}$. So $B$ must act trivially on $E$, i. e., there exist infinitely many $B$-orbits in $X_{2}$, a contradiction.

\section{REFERENCES}

[AB04] Valery Alexeev and Michel Brion, Stable reductive varieties. II. Projective case, Adv. Math. 184 (2004), no. 2, 380-408.

[Bat98] Victor Batyrev, Stringy Hodge numbers of varieties with Gorenstein canonical singularities, Integrable systems and algebraic geometry (Kobe/Kyoto, 1997), World Sci. Publ., River Edge, NJ, 1998, pp. 1-32.

[Bat99a] _ Birational Calabi-Yau n-folds have equal Betti numbers, New trends in algebraic geometry (Warwick, 1996), London Math. Soc. Lecture Note Ser., vol. 264, Cambridge Univ. Press, Cambridge, 1999, pp. 1-11.

[Bat99b] _ Non-Archimedean integrals and stringy Euler numbers of log-terminal pairs, J. Eur. Math. Soc. (JEMS) 1 (1999), no. 1, 5-33.

[Bit04] Franziska Bittner, The universal Euler characteristic for varieties of characteristic zero, Compos. Math. 140 (2004), no. 4, 1011-1032.

[BK94] Michel Brion and Friedrich Knop, Contractions and flips for varieties with group action of small complexity, J. Math. Sci. Univ. Tokyo 1 (1994), no. 3, 641-655.

[BM13] Victor Batyrev and Anne Moreau, The arc space of horospherical varieties and motivic integration, Compos. Math. 149 (2013), no. 8, 1327-1352.

[BP02] Michel Brion and Emmanuel Peyre, The virtual Poincaré polynomials of homogeneous spaces, Compositio Math. 134 (2002), no. 3, 319-335.

[Bri93] Michel Brion, Variétés sphériques et théorie de Mori, Duke Math. J. 72 (1993), no. 2, 369-404.

[Cra04] Alastair Craw, An introduction to motivic integration, Strings and geometry, Clay Math. Proc., vol. 3, Amer. Math. Soc., Providence, RI, 2004, pp. 203-225.

[DL99] Jan Denef and François Loeser, Germs of arcs on singular algebraic varieties and motivic integration, Invent. Math. 135 (1999), no. 1, 201-232.

[IS05] V. A. Iskovskikh and V. V. Shokurov, Birational models and flips, Uspekhi Mat. Nauk 60 (2005), no. 1(361), 29-98.

[Pas15a] Boris Pasquier, An approach of the minimal model program for horospherical varieties via moment polytopes, J. Reine Angew. Math. 708 (2015), 173-212. 
[Pas15b] _ A survey on the singularities of spherical varieties, arXiv:1510.03995v1.

[Pas16] , KLT singularities of horospherical pairs, Ann. Inst. Fourier (Grenoble) 66 (2016), no. 5, 2157-2167.

[Per14] Nicolas Perrin, On the geometry of spherical varieties, Transform. Groups 19 (2014), no. 1, 171-223.

[Rei83] Miles Reid, Decomposition of toric morphisms, Arithmetic and geometry, Vol. II, Progr. Math., vol. 36, Birkhäuser Boston, Boston, MA, 1983, pp. 395-418.

[Teh09] Jyh-Haur Teh, Motivic integration and projective bundle theorem in morphic cohomology, Math. Proc. Cambridge Philos. Soc. 147 (2009), no. 2, 295-321.

[Vey01] Willem Veys, Zeta functions and "Kontsevich invariants" on singular varieties, Canad. J. Math. 53 (2001), no. 4, 834-865.

Fachbereich Mathematik, Universität Tübingen, Auf der Morgenstelle 10, 72076 Tübingen, GERMAnY

E-mail address: batyrev@math.uni-tuebingen.de

Institut für Algebra, Zahlentheorie und Diskrete Mathematik, Leibniz Universität Hannover, Welfengarten 1, 30167 Hannover, Germany

E-mail address: gagliardi@math.uni-hannover.de 